\title{
PROKLA-Redaktion
}

\section{Editorial: Internationalisierung des Staates}

Die Krise der 1970er Jahre, die einen Strukturbruch in der ökonomischen Entwicklung der Nachkriegszeit markierte, war nicht nur eine Krise der Kapitalverwertung und der gesellschaftlichen Naturverhältnisse, sondern auch eine der politischen Herrschaft. Die neokonservativ-neoliberale Antwort war das Bemühen, die Ansprüche an die politische und demokratische Kontrollierbarkeit der gesellschaftlichen Entwicklung zu senken und die Regierungsfähigkeit für die herrschenden Gruppen der Gesellschaft wieder herzustellen. Im Zuge dieses Prozesses, der die Form der Globalisierung angenommen hat, kam es, wie von vielen Seiten beobachtet wurde, zu einer neuen Architektur politischer Herrschaft.

Die in der PROKLA vielfach analysierten ökonomischen, technologischen und ökologischen Prozesse veränderten auch die Stellung des kapitalistischen Staates im Verhältnis zu den kapitalistischen Produktionsverhältnissen. Es handelt sich nicht mehr, wie in den 1970er Jahren, um die Frage nach dem Verhältnis von Weltmarkt, Nationalstaat des kapitalistischen Zentrums und Imperalismus. Die kapitalistische Akkumulation vollzieht sich nicht mehr in nationalstaatlich getrennten und geschlossenen Räumen. Sie nimmt aufgrund der Schaffung eines globalen Finanzmarktes, zunehmenden ausländischen Direktinvestitionen und der fortschreitenden Transnationalisierung der Unternehmen und ihrer Produktionsketten sowie eines globalen Zugriffs auf die Arbeitskraft einen deterritorialisierten Charakter an. Ökonomische, politische und kulturelle Räume gliedern sich neu. Die historisch bezweifelbare Annahme, die Souveränität des Nationalstaats ermächtige diesen, seine Politik nach innen und außen zu verfolgen und durchzusetzen, erweist sich als wenig haltbar. Es waren wenige mächtige, imperialistische Staaten, die die ökonomische, politische, militärische und kulturelle Kapazität hatten, ihre Interessen nach innen und außen zur Geltung zu bringen. Angesichts einer neuen Formierung des globalen Raums kapitalistischer Ökonomie und Politik, angesichts intensiver ökonomischer, politischer, kulturell-kommunikativer und ökologischer Verflechtungen und Interdependenzen, die keineswegs gleichförmig sind, sondern neue Asymmetrien und imperiale Formen von Vorherrschaft und Abhängigkeit erzeugen, stellt sich seit einigen Jahren die Frage, ob es solche starken Nationalstaaten noch gibt, welche Handlungsmöglichkeiten staatliche Akteure haben, ob sie zunehmen oder sich schwächen. Die einen sehen die Souveränität des Nationalstaats ausgehöhlt, einen Verlust staatlicher Steuerungskompetenz, andere vermuten, dass es zu einer Stärkung des Staates gegenüber seinen Bürgern kommt, weil die Politik die ihr genehmen Entscheidungen auf dem Umweg über vermeintliche internationale Zwänge durchsetzen kann. Heide Gerstenberger diskutiert vor diesem Hintergrund die Leistungen, aber auch die Defizite der großen, kapitalismuskritischen Debattenstränge des 20. Jahrhunderts, in denen versucht wurde, den Staat als politische 
Form des Kapitalismus zu bestimmen. Die Frage nach der Souveränität des Staates und staatlich organisierter politischer Herrschaft stellt sich nicht nur sachlich im Gegenstandsbereich, sondern auch methodologisch. Seit einigen hundert Jahren dient in Europa der Nationalstaat als Modell für ein politisches Vokabular, in dessen Zentrum Begriffe wie Souveränität, Staat, Staatsvolk, Repräsentation, Demokratie stehen. Dieser Nationalstaat war auch schon lange vor dem Globalisierungsprozess nicht mehr der unbestrittene Leviathan, der über eine ungeteilte Souveränität verfügte. Ausfransungen, Überlagerungen und asymmetrische Abhängigkeiten politisch-staatlicher Mächte wurden seit dem 17. Jahrhundert immer wieder beobachtet. Dass das Zeitalter der Staatlichkeit zu Ende gehe, wurde von einem faschistischen Autor wie Carl Schmitt schon vor längerem behauptet, darüber sei kein Wort mehr zu verlieren. Dies lässt sich allerdings mit guten Argumenten bestreiten: dass die nationale Form des kapitalistischen Staates in die Krise geraten ist, heißt nicht, dass dieser selbst schon historisch überholt wäre. Es gibt gute Gründe, weiter über den Staat und die Staatlichkeit zu sprechen. Allerdings hat der "methodologische Nationalismus“ ausgedient. Der Nationalstaat steht nicht mehr allein im Zentrum der Analyse, er wird aber auch nicht einfach durch die Mächte der Triade USA, EU, Japan oder durch neue Imperien ersetzt; er scheint in seiner Bedeutung relativiert. Neue, vor allem internationale Instanzen und Herrschaftsagenturen sind neben ihn getreten und verändern die Mechanismen von Einflussnahme, Konsensbildung und Herrschaftsausübung. In diesen Prozessen drücken sich grundlegende Transformationen der Staatlichkeit aus, die im vorliegenden Heft der PROKLA als „Internationalisierung des Staates" gefasst werden. Tobias ten Brink zeigt in seinem Beitrag dass diese Internationalisierung, die sich in einer Vielzahl internationaler politischer Institutionen materialisiert, keineswegs, wie in vielen Ansätzen des politikwissenschaftlichen Mainstreams behauptet wird, zu einer Harmonisierung der immer stärker vernetzten Weltgesellschaft führt. Jens Wissel untersucht diese Internationalisierung und die damit einhergehenden Tendenzen zur Verselbständigung anhand einer besonders wichtigen Institution, der Welthandelsorganisation WTO. Auf einer grundsätzlichen, staatstheoretischen Ebene versuchen sich Ulrich Brand, Christoph Görg und Markus Wissen diesen neuen Entwicklungen zu nähern. In Anlehnung an Nicos Poulantzas entwickeln sie das Konzept der „Verdichtung zweiter Ordnung“. Poulantzas hatte den Staat als „Verdichtung“ von gesellschaftlichen Kräfteverhältnissen aufgefasst, womit er sowohl die Materialität der Institutionen ausdrücken wollte als auch den Sachverhalt, dass diese beständig umkämpft und im Wandel sind. Dass sich die Verhältnisse auf internationaler Ebene nochmals komplexer darstellen und dass auch die Defizite von Poulantzas' ursprünglichem Konzept in den Blick genommen werden müssen, wird in diesem Beitrag sehr deutlich aufgezeigt.

Bei der angesprochenen Internationalisierung des Staates handelt es sich um mehr als nur um einen Wandel des Staates. Metatheoretisch geht es darum, dass das Modell des Staates, wie er von Europa aus in den vergangenen Jahrhunderten im Zuge von Kolonialismus und Imperialismus und Neoimperialismus immer weiter verbreitet wurde, seine Gültigkeit verliert. Das Staatsvolk steht aufgrund der Dynamik von $\mathrm{Zu}$ - und $\mathrm{Ab}$ wanderung in Frage; das staatliche Territorium ist keineswegs fest umrissen, wenn der Staat die natürlichen Lebensgrundlagen wie Luft, Wasser oder Nahrung nicht kontrollieren kann; die staatliche Souveränität, die nach innen die Form der gesetzlich bindenden Selbsteinwirkung der Gesellschaft angenommen hat, wird unterhöhlt, wenn mächtige Akteure wie Kapitaleigner und Vermögensbesitzer, die durch Gesetz gebunden werden sollen, die Exit-Option wahr- 
nehmen oder zumindest damit drohen können.

Mehrere Trends lassen sich beobachten. Staatliche Akteure beziehen sich strategisch auf einen globalen Kontext, die Politik internationalisiert sich. Sie zielt wenig oder gar nicht darauf, nach Gleichheitsgesichtspunkten die Interessen aller BürgerInnen wahrzunehmen; in den Mittelpunkt rückt das Kriterium der Wettbewerbsfähigkeit, die von Unternehmen, Branchen, Regionen, Beschäftigtengruppen, Hochschulen und sozialstaatlichen Institutionen der sozialen Demokratie. Neu ist nicht der Wettbewerb als solcher, sondern das ihm nun auch die Bereiche des gesellschaftlichen Lebens unterworfen werden, die ihm bislang noch mehr oder weniger entzogen waren. Was sich im Wettbewerb als nachteilig erweisen könnte, wird zur Disposition gestellt. Das Handeln formell öffentlich-staatlicher Akteure verbindet sich mit privaten Akteuren zu Formen der Governance auf allen Ebenen der politischen Willensbildung und des Entscheidens. Wie in dem Aufsatz von Sol Picciotto herausgestellt wird, werden dabei die Grenzen zwischen öffentlichen und privaten Akteuren zunehmend verwischt. NGOs oder Unternehmen übernehmen staatliche Aufgaben, Unternehmensberater reorganisieren staatliche Institutionen nach marktwirtschaftlichen Gesichtspunkten, Gesetze werden von halböffentlichen Kommissionen oder gleich von Rechtsanwaltskanzleien verfasst und beschlossen und dem Parlament gerade noch zur Ratifizierung vorgelegt. Es kommt zu einer Denationalisierung, insofern politische Entscheidungen nach unten und oben verlagert werden. In der Geometrie politischer Macht wird über die Verknüpfung der politischen Institutionen und die räumliche Reichweite politischen Entscheidens selbst flexibel entschieden: Dezentralisierung staatlicher Kompetenzen; Kommunen oder Bundesländer, die bei der Europäischen Kommission direkt verhandeln; den Nationalstaat bindende Entscheidungen in intergouvernementa- len Organisationen - das alles ist an der Tagesordnung. Die Frage, der dieses Heft der PROKLA nachgeht, ist, wie weit solche Prozesse der Internationalisierung und Denationalisierung reichen.

Für die Staaten der Peripherie stellen sich diese Prozesse jedenfalls anders dar als für Staaten des kapitalistischen Zentrums. Miriam Heigl wirft in ihrem Text die Frage auf, wie Staatlichkeit in peripheren Formationen überhaupt zu analysieren ist. In ihrem Beitrag kommt sie zu dem Ergebnis, dass hier weder die Dependenztheorie noch das Konzept der „failed states“ wirklich weiterhelfen, sondern eher eine an den staatstheoretischen Überlegungen von Gramsci und Poulantzas ausgerichtete Konzeptualisierung, wie am Beispiel von Mexiko verdeutlicht wird. Die Vorstellung von „failed states“, von „entgrenzten“ und „asymmetrischen" Kriegen spielt auch in Herfried Münklers Rechtfertigung imperialer Politik eine bedeutende Rolle. Raul Zelik setzt sich kritisch mit Münklers Überlegungen auseinander; er zeigt nicht nur deren Unzulänglichkeiten auf, sondern auch deren Funktionalität für die neuen, meistens in europäischem Gewand auftretenden Machtansprüche der Berliner Republik.

Für die Staaten des kapitalistischen Zentrums lassen sich die Tendenzen zur Internationalisierung gut anhand der G 8Treffen diskutieren; es handelt sich um ein Treffen der Regierungschefs der wirtschaftlich und politisch mächtigsten Staaten. Die Treffen hatten anfangs, also Mitte der 1970er Jahre, jedoch einen beinahe privaten Charakter, es ging darum, sich über gemeinsame Probleme auszutauschen. Stefan Schoppengerd zeigt in seinem Beitrag, wie im Laufe der Zeit die G 8-Treffen zu einem bedeutenden Governance-Mechanismus heranwuchsen. Es kam zu Treffen von Fachministern, es wurden Arbeitsgruppen von Experten zu einzelnen Fragen eingerichtet. Aus diesen Diskussionsprozessen entstanden im Weiteren zahlreiche Politikkonzepte und Strategien. Die G 8-Treffen erweisen sich 
als ein Baustein einer neuen Form transnationalen Regierens jenseits formeller Instanzen und Regelungen. Wie das einzuschätzen ist, ist keineswegs klar. Doch hat die Antwort Konsequenzen auch für alle jene kapitalismuskritischen Kräfte, die das Ziel einer demokratischen Form der Globalisierung, einer nachhaltigen und solidarischen Produktionsform, eines Lebens ohne Sexismus und Rassismus verfolgen. Sie alle sehen sich mit mehreren Möglichkeiten konfrontiert: Sollen sie sich in ihren politischen Kämpfen und Forderungen immer noch vorwiegend auf den jeweiligen Nationalstaat konzentrieren und an die jeweiligen politischen Vertreter herrschender Interessen wenden? Sollen sie sich gegen die intergouvernementalen und informellen Treffen wenden, weil dort über globale Herrschaftskonzepte gesprochen wird und sich hier besonders mächtige Einzelstaaten wie die USA behaupten? Geht es also darum, die nationalen Repräsentanten unter Druck zu setzen, dass sie nicht klein beigeben und den nationalstaatlichen Raum gegenüber den Zugriffen imperialer Mächte abschirmen? Schließlich ist $\mathrm{zu}$ fragen, ob nicht $\mathrm{G}$ 8-Treffen, WTO, IWF, Rating-Agenturen, einzelne nationale Staatsapparate, Zentralbanken gemeinsam ein komplexes Netzwerk des globalen Regierens bilden, das deswegen auch Ziel eines eigenständigen Widerstands darstellt. Dies sind Fragen, auf die die Beiträge noch keine endgültigen Antworten geben können. Aber sie stellen zentrale Probleme und Aspekte einer Diskussion dar, die geführt wird, und die in großer Dringlichkeit in den nächsten Jahren noch weiter zu führen sein wird.

Zum Schwerpunkt dieses Heftes hat Ulrich Brand nicht nur (gemeinsam mit Christoph Görg und Markus Wissen) einen Artikel beigesteuert; als Gastredakteur war er auch maßgeblich an der Konzeption und am Zustandekommen dieser Ausgabe beteiligt, wofür wir ihm herzlich danken.

\section{PROKLA 148 (September 2007): Die „Verbetriebswirtschaftli- chung" der Gesellschaft: Soziale Praxen, Widersprüche und Widerstand}

In den letzten Jahren konnte man nicht nur in Deutschland eine zunehmende Ökonomisierung aller Bereiche des gesellschaftlichen Lebens beobachten. So wird versucht in Institutionen, wie Behörden, Krankenhäuser oder Universitäten, die bisher der Logik des Marktes nur begrenzt unterworfen waren, ein profitorientiertes Handeln durchzusetzen; das „betriebswirtschaftliche“ Denken wird zum anscheinend allgemeingültigen Leitbild. Aber auch die kapitalistischen Unternehmen selbst werden umgestaltet. Beim Versuch sämtliche Ressourcen der Ware Arbeitskraft für den kapitalistischen Verwertungsprozess zu mobilisieren, werden neue Unternehmenskulturen eingeführt, die Selbstverantwortung und Anerkennung vermitteln sollen. Vor allem qualifizierte Beschäftigte werden damit auch emotional an ihren Arbeitsplatz gebunden, während das private Leben unter Zeitdruck „organisiert" werden muss. Das Heft will die Widersprüche und sozialen Praxen in den Blick nehmen, die 'vor Ort' durch die veränderten Rahmensetzungen 'von oben' entstehen. Denn im Zuge der „Verbetriebswirtschaftlichung“ werden Machtverhältnisse vor Ort neu verhandelt, veränderte Herrschaftspraktiken etabliert, Mechanismen erfunden, die vielfach erst die Märkte und Pseudomärkte produzieren, auf deren Druck sie vermeintlich reagieren. 